\section{Attention in the pigeon: Novelty effects and testing with compounds*}

THOMAS ZENTALL

University of Pittsburgh, Pittsburgh, Pa. 15213

Farthing \& Hearst (1970) have shown that following a successive discrimination between a vertical line on a blue background ( $\mathrm{S}+$ ) and a horizontal line on a green background $(S-)$, pigeons tested with the line-tilt elements alone showed little responding to either value, but when placed on a blue background, differential line-tilt control was observed. The present experiment asked whether this control could have been a function of differential compound novelty (blue-horizontal is novel, while blue-vertical is not). Pigeons received single-stimulus pretraining with a red field prior to discrimination training. When tested with the novel compounds, red-vertical and red-horizontal, differential responding was found, indicating that compound novelty could not account for the observed line-tilt control.

Reynolds (1961) trained two pigeons to discriminate between successive presentations of a white triangle on a red background (S+) and a white circle on a green background (S-). When each of the four stimulus elements was tested in isolation, one of the birds responded almost entirely to the red background, while the other bird made virtually all of its responses to the white triangle (on a black background). Reynolds concluded that "... a pigeon may attend to only one of several aspects of a discriminative stimulus." Reynolds defines attention in terms of the ability of a dimension to exert stimulus control over responding. Thus, one of the birds appeared to attend to color, while the other attended to shape. Farthing \& Hearst (1970) point out, however, that since responding was virtually absent to both values of the "weaker" dimension, stimulus control may be obscured by a floor effect. They maintain that in order to evaluate stimulus control, one needs to find a nonzero response rate to at least one value on the dimension, and they propose that by combining the positive stimulus from the more powerful dimension with both values from the less powerful dimension, one would have a response baseline against which to measure stimulus control. As part of a larger study, they trained four birds to discriminate between a white vertical line on a blue background (S+) and a white horizontal line on a green background (S-). Tested in extinction, the birds were presented not only with the

*Supported by Grant FR07084 from the National Institutes of Health to the University of Pittsburgh and by Grant MH19757 from the National Institute of Mental Health to the author. The author wishes to thank David Hogan for his help in running experimental sessions. stimulus elements-blue, green, vertical, and horizontal-but also with the various compounds-blue-vertical, blue-horizontal, green-vertical, and green-horizontal. All birds showed stimulus control of responding along the color dimension, while virtually no responses were made to either the vertical or horizontal stimulus by any of the birds. On the other hand, when the line stimuli were presented on a blue background, large differences in responding between the blue-vertical and blue-horizontal compounds were observed, indicating control by the line-tilt dimension. Thus, it appears that there was stimulus control by the line-tilt dimension in spite of a virtual absence of responding to either line-tilt alone.

An alternative interpretation of the Farthing and Hearst results involves the relative novelty of the two stimulus compounds, blue-vertical and blue-horizontal. The only test stimuli with which the birds had had experience during training were the blue-vertical and the green-horizontal compounds. All the other test stimuli were novel, in the sense that they were not presented in the same form during training. They were either individual elements of the training compounds (i.e., vertical) or new compounds made up by pairing an element from one training compound with an element from the other training compound (i.e., blue-horizontal). The observed differential responding to blue-vertical (a training compound) and blue-horizontal (a novel compound) may be a measure of differential novelty rather than an indication of differential control along the line-tilt dimension.

The present experiment attempted to test the novel stimulus interpretation of the Farthing and Hearst data by preceding the discrimination training by single
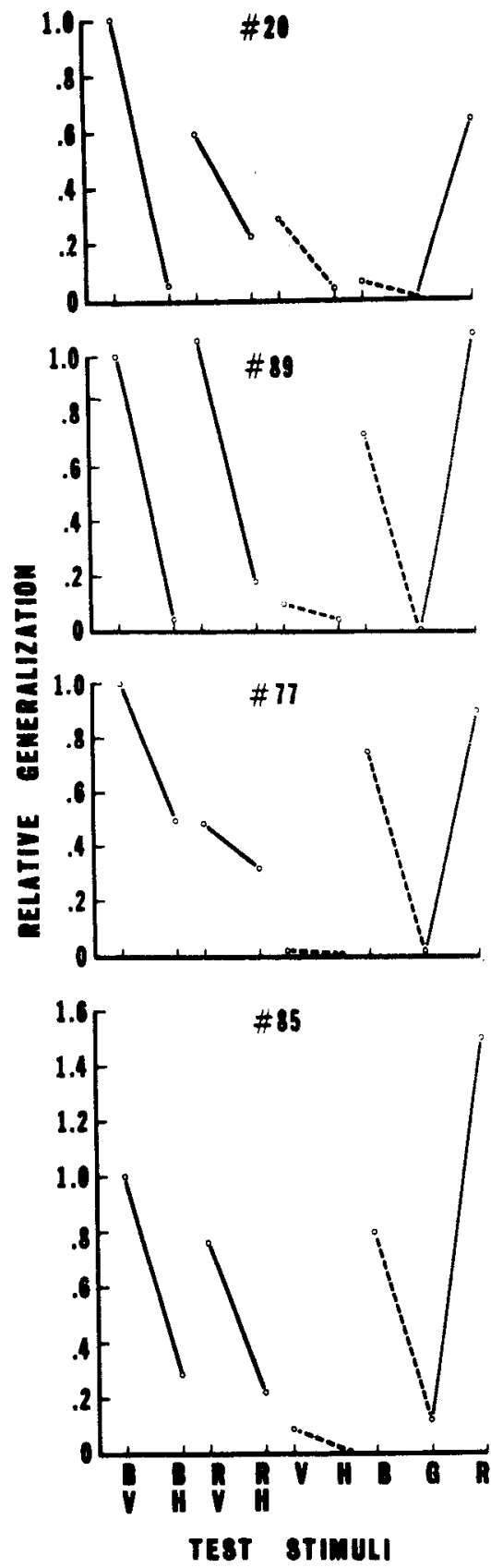

Fig. 1. Relative responding during test session. $B=$ blue field, $G=$ green field, $R=$ red field, $V=$ white vertical line on black field, $H=$ white horizontal line on black field, BV = white vertical line on blue field, $B H=$ white horizontal line on blue field, RV $=$ white vertical line on red field, and RH = white horizontal line on red field.

stimulus training with a plain red background. During the extinction test, responses to the novel compounds, red-vertical and red-horizontal, provided a means of measuring stimulus control, uncontaminated by differential 
novelty effects, since both stimulus compounds involving the red background were equally novel.

\section{SUBJECTS}

Four female experimentally naive White Carneaux pigeons, approximately 1 year old, were maintained at $80 \%$ of their free-feeding body weights. APPARATUS

The experimental box was a standard single response-key pigeon test chamber (Lehigh Valley Electronics No. 1519) with an in-line readout projector (IEE Series 10) mounted behind the key. Any of the following stimuli could be projected on the key: a $3.5 \times 25.4 \mathrm{~mm}$ white vertical line on a black background (V), a white horizontal line on a black background $(\mathrm{H})$, a plain blue field (B) produced by Kodak Wratten Filter No. 38A, a plain green field (G) produced by Kodak Wratten Filter No. 60, and a plain red field (R) produced by Kodak Wratten Filter No. 26, as well as any combination of color and line together [i.e., a white vertical line on a blue background (BV)]. A dim houselight was turned on during all stimulus presentations. Reinforcement consisted of $5 \mathrm{sec}$ access to mixed grain (Purina Pigeon Grain). Masking of external sounds was provided by white noise and a blower fan. Control equipment was located in an adjoining room.

$$
\text { PROCEDURE }
$$

Single Stimulus Training

All birds were magazine trained, shaped to peck the red response key, and then given 30 continuous reinforcements, each day, on the first 2 days of training. On the third day, the first of six sessions began in which responses were reinforced on a 1 -min variable interval schedule of reinforcement (VI $1 \mathrm{~min}$ ). During the first five of these sessions, $R$ was presented for 6030 -sec periods, each separated by a 10-sec blackout. During blackout periods, the key was dark, the houselight was off, and responses were ineffective. The sixth session differed from the first five in that BV was the training stimulus.
Discrimination Training

Following single stimulus training, the birds received six discrimination training sessions, involving $\mathrm{BV}(\mathrm{S}+)$ and $\mathrm{GH}(\mathrm{S}-)$. Discrimination sessions consisted of 30 presentations of $\mathrm{S}+$ semirandomly alternated with 30.5 presentations. Responses to $\mathrm{S}+$ were reinforced on a VI 1-min schedule; responses to $\mathrm{S}-$ were extinguished. All presentations were for $30 \mathrm{sec}$ separated by 10 -sec blackouts.

$$
\text { Testing }
$$

A single test session followed the last day of discrimination training. Presentation of test stimuli was preceded by a brief warmup period involving five presentations each of $\mathrm{S}+$ and $S-$ with the training contingencies in effect. The test stimuli presented were: $B, G, R, V, H, B V, B H, R V$, and $\mathrm{RH}$. Testing consisted of 30 -sec presentations of the nine test stimuli in 12 randomized permutations, with stimulus presentations separated by 10 sec of blackout, as during training. Responses during the test presentations were not reinforced.

\section{RESULTS}

The discrimination ratio (total responses to $\mathrm{S}-$ divided by total responses to $\mathrm{S}^{+}$) on the last day of discrimination training was below .04 for all birds.

The test data for the individual birds are prosented in Fig. 1. Responding to each of the test stimuli $h$ as been indicated relative to responding to $\mathrm{BV}$. Differences in responding among the stimulus elements present curing discrimination training indicate that, for three of the birds, the wavelength dimension controlled responding more than did the line-tilt dimension. For the fourth bird, neither dimension exerted great control, though more control by the line-tilt dimension was found. In contrast to single-element responding, clear evidence for line-tilt control was found for all birds when responding to $\mathrm{BV}$ and $\mathrm{BH}$ were compared. Control along the line-tilt dimension was also indicated by the difference in responding between $\mathrm{RV}$ and $\mathrm{RH}$. For all four birds, the gradients along the line-tilt dimension were steepened by the addition of either a blue or a red background

\section{DISCUSSION}

The results support and extend the Farthing and Hearst findings. Near-zero responding along a stimulus dimension may obscure differential control along the dimension. To observe this control, one can present values along the dimension in compound with a stimulus which is known to produce responding. Also, one can conclude that the line-tilt control found with compound stimuli $\mathrm{BV}$ and $\mathrm{BH}$ could not be explained by differences in compound novelty, for line-tilt control was also found when both compounds ( $\mathrm{RV}$ and $\mathrm{RH}$ ) were equally novel. There is some suggestion that novelty may have been responsible for some of the differences observed between blue-vertical and blue-horizontal responding. Two of the four birds showed much flatter line-tilt gradients when they were presented against a red background than when presented against a blue background. For the other two birds, the difference was minimal but in the same direction. Also, two of the birds showed appreciably more responding to red than to red-vertical, while for none of the birds was the reverse true. It may be, however, that summation effects with the red-vertical compound are partially obscured due to the fact that the addition of vertical to red removes some of the red area. The reduction in response tendency produced by altering the red stimulus may be greater than the increase in response tendency produced by the addition of vertical. In order to test for a novelty effect, one needs to use dimensions which can be presented together without one partially obscuring the other.

\section{REFERENCES}

FARTHING, G. W., \& HEARST, E. Attention in the pigeon: Testing with compounds or elements. Learning \& Motivation, 1970, 1, 65-78.

REYNOLDS, G. S. Attention in the pigeon. Journal of the Experimental Analysis of Behavior, 1961, 4, 203-208. 